\title{
Automating BioSense 2.0 Locker Processing for Local Program-Specific Surveillance
}

\author{
Harold Gil*, Jeffrey Johnson, Brit Colanter and Jessica Yen \\ County of San Diego, Health and Human Services Agency, Public Health Services, San Diego, CA, USA
}

\section{Objective}

The purpose of this project is to develop a capacity to facilitate implementation of a user-driven enhanced process for generating program-specific surveillance reports from BioSense 2.0 locker data.

\section{Introduction}

Syndromic surveillance can provide early warning of potential public health emergencies and acute health events in a population. The sharing and aggregation of syndromic data among jurisdictions can provide more comprehensive situational awareness and improve coordination and decision-making. The BioSense 2.0 Program supports increased syndromic data-sharing among a nationwide network of local and state public health agencies. Most users of this application utilize the main web site front-door interface due to its user-friendly features for query and analysis. However, this interface currently has a limited number of analytic tools, export functions, and provides access only to binned data. The back-door interface, with access to additional data lockers containing raw and exception data, represents a potentially rich source of untapped and underutilized information. In this presentation, we discuss our ongoing development and early success of a capacity (consisting of code libraries, a parser, and an implementation guide) that allows users to tailor a program-specific, automated process for generating surveillance reports from their BioSense 2.0 data locker. The product will soon be available to members of the BioSense User Community.

\section{Methods}

Progress has been made in developing a collection of materials we refer to as a "package" comprising sample query and analysis documented-code libraries for SQL, SAS and R, a chief complaint parser, and an implementation guide. We are soliciting input from members of the BioSense User Community during the current development phase of this package to ensure that the programmed functions in the code libraries address common needs across jurisdictions. We have begun to apply this package to our local syndromic surveillance data at San Diego County Public Health Services to demonstrate package reliability and proof-of-concept. Our local objectives are to access the locker data to perform quality assurance, to export data for more detailed analysis, and to supplement our local syndromic surveillance system with data from several hospitals connected to BioSense.

\section{Results}

Working with several jurisdictions within the BioSense User Community, the concept of a package with the purpose described above has been well-received. A number of BioSense community users have collaborated to help define a set of basic functions and capabilities that cover common surveillance needs across public health agencies. Preliminary outcomes have shown that this package is capable of producing results identical to at least a few query and analysis results obtained from the front-door interface, suggesting appropriate behavior by the package. Several users have tested the package and found the new functions useful for quality assurance purposes.

\section{Conclusions}

The BioSense 2.0 front-door interface offers user-friendly tools for query and analysis of syndromic surveillance data. However, the current front-door interface has limited data access and defined query and analytic capabilities. Based on open discussions with BioSense users and their participation in input discussions for the development of the package, there is an unmet demand for more robust query and analytic capabilities from BioSense 2.0, additional documentation of the BioSense locker function and potential, as well as the type of data to which these can be applied. Advantages offered by use of the package include automation and greater query and analytic versatility and flexibility compared to the current BioSense 2.0 front-door user interface.

\section{Keywords}

BioSense; user community; automate; code libraries

\section{Acknowledgments}

This work was supported in part by an appointment to the Applied Public Health Informatics Fellowship Program administered by CSTE and funded by the Centers for Disease Control and Prevention (CDC) Cooperative Agreement 3U38HM000414-04W1.

\author{
*Harold Gil \\ E-mail: harold.gil@sdcounty.ca.gov
}

